J. Clin. Chem. Clin. Biochem.

Vol. 19, 1981, pp. 453-456

\title{
Comparison of Eleven Commercially Available Kits for the Radioimmunoassay of Serum Thyroxine ${ }^{1}$ )
}

\author{
By L. H. Elvers, J. C. Koedam and J. G. Loeber
}

National Institute of Public Health, Bilthoven, The Netherlands

(Received March 7, 1980/February 2, 1981)

Summary: Eleven of the thyroxine RIA kits commercially available in the Netherlands were compared with respect to reliability, sensitivity, simplicity and performance. The intra- and interassay coefficients of variation ranged from 4.6 to $11.3 \%$, whereas the recovery of added thyroxine ranged from 85 to $114 \%$. The labour-time per 100 tubes varied from 115 to 175 minutes.

\section{Vergleich von elf kommerziell erhältlichen Bestecks für den Radioimmunassay von Thyroxin im Serum}

Zusammenfassung: Elf der in den Niederlanden kommerziell erhältlichen Testbestecks für den Radioimmunassay von Thyroxin wurden hinsichtlich Zuverlässigkeit, Empfindlichkeit und Einfachheit der Ausführung verglichen. Die Variationskoeffizienten in der Serie und von Tag zu Tag waren im Bereich von 4,6-11,3\% und die Wiederfindung von zugesetztem Thyroxin betrug 85-114\%. Hundert Ansätze können in 2-3 Stunden bearbeitet werden.

\section{Introduction}

One of the parameters for the evaluation of thyroid function is the serum thyroxine $\left(\mathrm{T}_{4}\right)$ level. During recent years many manufacturers have introduced kits for the radioimmunoassay of serum $T_{4}$. This study was undertaken to make an inventory of a number of objective criteria with the aim of facilitating the choice of kit.

\section{Materials and Methods}

\section{Reagents}

The following kits were used and further referred to as mentioned in parentheses:

1. T4-RIA-PEG; Abbott Laboratories, North Chicago, Ill. U.S.A. (Abbott),

2. $T_{4}$ Solid Phase RIA; Becton Dickinson Immunodiagnostics, Orangeburg, NY, U.S.A. (Becton D),

3. Riamat $T_{4}$; Byk Mallinckrodt, Dietzenbach-Steinberg, Germany (Byk Riamat),

4. SPAC $T_{4}$; Byk Mallinckrodt, Dietzenbach-Steinberg, Germany (Byk Spac $\mathrm{T}_{4}$ ),

5. Gamma Coat $T_{4}$; Clinical Assays, Cambridge, Ma. U.S.A. (Clinịcal Assays),

1) Reference to a company and/or product is only for the purpose of information and identification and does not imply approval or recommendation of the company and/or product by the National Institute of Public Health to the exclusion of others which may also be suitable.
6. Immophase $\mathrm{FT}_{4}-\mathrm{RIA}^{2}$ ); Corning Medical, Midfield, Ma, U.S.A. (Corning),

7. T4-RIA-Premix; Diagnostic Products Corporation, Los Angeles, Calif., U.S.A. (DPC-Premix),

8. $\mathrm{T}_{4}$-RIA-Autopak; Micromedic Systems, Horsham, Pa, U.S.A. (Micromedic),

9. Tetratab RIA $T_{4}$, Nuclear Medical Laboratories, Dallas, Tx., U.S.A. (NML),

10. $\mathrm{T}_{4}$-RIA IM 801, The Radiochemical Centre, Amersham, U.K. (RCA IM 801),

11. T4-RIA PEG IM 921; The Radiochemical Centre, Amersham, U.K. (RCA IM 921).

$L$-thyroxine (T-2376) was purchased from Sigma (St. Louis, Miss., U.S.A.). All other chemicals were reagent grade mainly from Merck (Darmstadt; Germany).

\section{Sera}

Sera from hypothyroid (C2), euthyroid $(C 4, C 5)$ and hyperthyroid patients $(\mathrm{C7}-\mathrm{C10})$ were obtained from the Central Blood Bank, Amsterdam, the General Zeister Hospital, Zeist and the Antonius Hospital, Utrecht. The sera C3 and C6 were prepared by pooling of sera with various $\mathrm{T}_{4}$ levels.

\section{Procedure}

For the determination of the intra- and interassay variation for each kit four experiments were carried out, two with one kit specimen and two with another, originating from a different batch. In each experiment the nine sera $\mathrm{C} 2-\mathrm{C} 1 \mathrm{Q}$, were measured in triplicate. For the determination of the percentage recovery in

2) The Corning Immophase $\mathrm{FT}_{4}$-RIA, although meant for the determination of free serum $T_{4}$ also yields a value for total serum $\mathbf{T}_{\mathbf{4}}$. 
a fifth experiment, 75,150 and $225 \mathrm{nmol} / 1 \mathrm{~T}_{4}$ were added to $T_{4}$ free serum, which had been prepared as follows:

$16 \mathrm{~g}$ charcoal was added to $100 \mathrm{ml}$ serum; after mixing for $20 \mathrm{~h}$ at $4^{\circ} \mathrm{C}$ the suspension was centrifuged for $60 \mathrm{~min}$ at $7000 \mathrm{~g}$; the serum was decanted and centrifuged for $60 \mathrm{~min}$ at $13000 \mathrm{~g}$; finally the serum was filtered through a $0.22 \mu \mathrm{m}$ millipore filter.

\section{Results}

Table 1 shows technical data from each kit, such as the way in which $\mathrm{T}_{4}$ is liberated from the binding proteins, the number of standards, with matrix, incubation time and incubation temperature, separation method and the total amount of radioactivity contained.

The intra- and interassay coefficients of variation (tab. 2) for the sera $\mathrm{C}_{2}-\mathrm{C}_{10}$ were calculated as outlined by McDonagh et al. (1).

In table 3 the mean $T_{4}$ concentrations of each serum sample as measured by all kits are presented. To determine whether any kit systematically yields high or low
$\mathrm{T}_{4}$ values all kits were ranked from 1 to 11 according to their results for each serum sample. The kit yielding the lowest $T_{4}$ value for serum $C 2$ wass given number 1 , the kit yielding the next higher value was given number 2 etc. The same procedure was followed for the other sera except serum C9, since this serum did not fall within the measuring range for all kits. For each kit the sum of ranking numbers was calculated by adding the individual numbers. According to Thompson \& Willke (2) the "critical values" for the sum of ranking numbers for 8 sera and 11 kits are 23 and 73 (with $95 \%$ confidence level). According to these figures one kit, Byk-Riamat, measures significantly low values, whereas three kits (Abbott, Becton D and NML) measure too high.

The percentage recovery of known amounts of $T_{4}$ added to $T_{4}$-free serum as measured by each kit is presented in table 4. For the kits with no zero control sample the response of the $T_{4}$-free serum was much lower than that of the first standard point. For the kits containing a zero sample there was no significant difference in response

Tab. 1. Technical data of $T_{4}$ RIA kits.

\begin{tabular}{|c|c|c|c|c|c|c|c|c|}
\hline Kit & $\begin{array}{l}\text { Liberation of } \\
\mathrm{T}_{4} \text { from TBG/ } \\
\text { albumin }\end{array}$ & $\begin{array}{l}\text { Star } \\
\text { rang } \\
\text { no. }\end{array}$ & $\begin{array}{l}\text { dards } \\
\text { e: nmol/1 } \\
\text { - matrix }\end{array}$ & $\begin{array}{l}\text { Sample } \\
\text { volume } \\
(\mu \mathrm{l})\end{array}$ & $\begin{array}{l}\text { Incubation } \\
\text { time } \\
\text { (min) }\end{array}$ & $\begin{array}{l}\text { Incubation } \\
\text { temp. } \\
\left.{ }^{\circ} \mathrm{C}\right)\end{array}$ & $\begin{array}{l}\text { Separation } \\
\text { method }\end{array}$ & $\begin{array}{l}{\left[{ }^{125} \mathrm{I}\right] \mathrm{T}_{4}} \\
\text { per } 100 \\
\text { tubes }(\mathrm{kBq})\end{array}$ \\
\hline Abbott & $\begin{array}{l}\text { ANS + Thiomersal } \\
\text { Barbital }\end{array}$ & $\begin{array}{l}0 \\
5\end{array}$ & $\begin{array}{l}-309 \\
- \text { AS }\end{array}$ & 25 & $\begin{array}{l}60^{1} \\
120\end{array}$ & $\begin{array}{l}37 \\
\text { RT }\end{array}$ & PEG & $<166$ \\
\hline Becton D & $\begin{array}{l}\text { ANS } \\
\text { Barbital }\end{array}$ & $\begin{array}{l}0 \\
6\end{array}$ & $\begin{array}{l}-309 \\
- \text { HS }\end{array}$ & 10 & $\begin{array}{l}45^{1} \\
50\end{array}$ & $\begin{array}{l}37 \\
\text { RT }\end{array}$ & ACT & $<333$ \\
\hline Byk-Riamat & $\begin{array}{l}\text { ANS } \\
\text { Barbital }\end{array}$ & $\begin{array}{l}0 \\
7\end{array}$ & $\begin{array}{l}-515 \\
-H S\end{array}$ & 10 & $\begin{array}{l}30 \\
60^{1}\end{array}$ & $\begin{array}{l}37 \\
\text { RT }\end{array}$ & $\begin{array}{l}\text { ion-exchange } \\
\text { resin }\end{array}$ & $<148$ \\
\hline Byk-Spac $T_{4}$ & $\begin{array}{l}\text { ANS } \\
\text { Barbital }\end{array}$ & $\begin{array}{l}0 \\
7\end{array}$ & $\begin{array}{l}-515 \\
-\mathrm{HS}\end{array}$ & 25 & $\begin{array}{l}60^{1} \\
120\end{array}$ & $\begin{array}{l}37 \\
\text { RT }\end{array}$ & ACT & $<148$ \\
\hline Clinical Assays & $\begin{array}{l}\text { ANS } \\
\text { Na-salicylate }\end{array}$ & $\begin{array}{l}0 \\
6\end{array}$ & $\begin{array}{l}-386 \\
-\mathrm{HS}\end{array}$ & 10 & 45 & RT & $\mathrm{ACT}$ & $<185$ \\
\hline Corning & Thiomersal & $\begin{array}{r}13 \\
5\end{array}$ & $\begin{array}{l}-315 \\
-\mathrm{HP}\end{array}$ & 25 & $20+30$ & RT & $\begin{array}{l}\text { immiuno- } \\
\text { sorbent }\end{array}$ & $<247$ \\
\hline DPC-Premix & $\begin{array}{l}\text { ANS } \\
\text { Barbital }\end{array}$ & $\begin{array}{l}0 \\
6\end{array}$ & $\begin{array}{l}-309 \\
- \text { AS }\end{array}$ & 25 & $\begin{array}{l}20 \\
30^{1}\end{array}$ & $\begin{array}{l}\mathrm{RT} \\
50\end{array}$ & $\begin{array}{l}\text { 2nd antibody } \\
\text { body + PEG }\end{array}$ & $<222$ \\
\hline Micromedic & $\begin{array}{l}\text { ANS } \\
\text { Barbital }\end{array}$ & $\begin{array}{l}0 \\
7\end{array}$ & $\begin{array}{l}-412 \\
-\mathrm{HP}\end{array}$ & 20 & 60 & 37 & ACT & $<370$ \\
\hline NML & $\begin{array}{l}0.025 \mathrm{~mol} / 1 \mathrm{HCl} \\
\text { Barbital }\end{array}$ & $\begin{array}{r}15 \\
5\end{array}$ & $\begin{array}{l}-304 \\
- \text { HS/AS }\end{array}$ & 10 & $\begin{array}{l}30-60 \\
60^{1}\end{array}$ & $\begin{array}{l}\text { RT } \\
\text { RT }\end{array}$ & $\begin{array}{l}\left(\mathrm{NH}_{4}\right)_{2} \mathrm{SO}_{4} \\
\text { precipitation }\end{array}$ & $<370$ \\
\hline RCA-IM 801 & Thiomersal & $\begin{array}{l}6.4 \\
4\end{array}$ & $\begin{array}{l}-275 \\
-\mathrm{HS}\end{array}$ & 50 & 60 & 37 & $\begin{array}{l}\text { Adsorption } \\
\text { powder }\end{array}$ & $<444$ \\
\hline RCA-IM 921 & Thiomersal & $\begin{array}{r}12 \\
4\end{array}$ & $\begin{array}{l}-275 \\
- \text { HS }\end{array}$ & 50 & 45 & $\mathbf{R T}$ & PEG & $<444$ \\
\hline
\end{tabular}

ANS = 8-anilino-1-naphthalene sulphonic acid;

AS = animal serum;

HS = human serum;

HP = human plasma;

PEG = polyethyleneglycol $\left(M_{\mathrm{T}}=6000\right)$;

ACT = antibody-coated tube;

$\mathrm{RT}=$ room temperature.

1 denotes the conditions chosen here in the case of several prescribed possibilities. 
Tab. 2. The intra- and interassay coefficients of variation for 11 thyroxine kits.

\begin{tabular}{|c|c|c|c|c|c|c|c|c|c|}
\hline \multirow{2}{*}{ Kit/ Serum } & \multicolumn{9}{|c|}{$\mathrm{CV}^{1}$ intraassay $(\%)$} \\
\hline & $\mathbf{C 2}$ & C3 & C4 & C5 & $\mathrm{C} 10$ & $\mathrm{C} 6$ & $\mathrm{C7}$ & $\mathrm{C} 8$ & $\mathrm{C} 9$ \\
\hline \multirow{12}{*}{$\begin{array}{l}\text { Abbott } \\
\text { Becton D } \\
\text { Byk-Riamat } \\
\text { Byk Spac T } 4 \\
\text { Clinical Assay } \\
\text { Corning } \\
\text { DPC-Premix } \\
\text { Micromedic } \\
\text { NML } \\
\text { RCA IM } 801 \\
\text { RCA IM } 921\end{array}$} & 7.5 & 4.6 & 3.2 & 2.5 & 2.1 & 2.3 & 1.1 & 2.5 & 4.0 \\
\hline & 5.2 & 3.6 & 4.6 & 3.2 & 5.3 & 7.5 & 6.2 & 5.4 & 3.1 \\
\hline & 14.3 & 8.3 & 7.9 & 4.9 & 9.4 & 13.6 & 4.6 & 8.2 & 5.1 \\
\hline & 3.8 & 3.9 & 6.4 & 3.1 & 4.2 & 3.7 & 3.3 & 1.4 & 3.2 \\
\hline & 10.6 & 7.2 & 5.6 & 3.0 & 4.0 & 3.3 & 3.0 & 4.0 & 3.2 \\
\hline & 3.8 & 1.8 & 3.3 & 3.5 & 4.1 & 2.1 & 2.4 & 1.5 & 2.5 \\
\hline & 2.9 & 3.0 & 3.1 & 3.8 & 2.9 & 2.0 & 3.4 & 3.1 & 5.4 \\
\hline & 6.4 & 4.7 & 5.4 & 5.2 & 4.2 & 3.5 & 3.8 & 4.9 & 4.2 \\
\hline & 6.1 & 3.8 & 2.4 & 2.5 & 2.8 & 4.7 & 5.2 & 3.0 & 3.8 \\
\hline & 2.0 & 2.9 & 1.4 & 1.8 & 1.5 & 2.3 & 2.4 & 1.7 & 1.8 \\
\hline & 5.2 & 4.1 & 2.0 & 2.6 & 1.8 & 3.0 & 4.0 & 4.0 & 2.6 \\
\hline & \multicolumn{9}{|c|}{$\mathrm{CV}^{2}$ interassay $(\%)$} \\
\hline Abbott & 8.9 & 4.6 & 7.6 & 4.3 & 4.8 & 3.8 & 2.5 & 2.9 & 4.0 \\
\hline Becton D & 15.7 & 13.1 & 9.5 & 10.3 & 9.6 & 8.0 & 9.3 & 9.0 & 5.9 \\
\hline Byk-Riamat & 19.2 & 10.9 & 7.9 & 8.1 & 9.4 & 13.6 & 6.6 & 9.2 & 5.1 \\
\hline Byk Spac $\mathrm{T}_{4}$ & 6.3 & 3.9 & 6.7 & 7.8 & 8.3 & 3.7 & 6.6 & 4.5 & 5.6 \\
\hline Clinical Assays & 15.5 & 9.7 & 8.9 & 9.1 & 6.0 & 7.3 & 9.6 & 8.5 & 7.2 \\
\hline Corning & 13.0 & 3.2 & 4.4 & 4.5 & 4.7 & 2.2 & 2.4 & 3.8 & 7.3 \\
\hline DPC-Premix & 5.1 & 6.5 & 5.5 & 5.8 & 6.2 & 5.6 & 7.0 & 11.2 & 14.1 \\
\hline Micromedic & 9.4 & 9.1 & 10.6 & 10.6 & 6.4 & 6.6 & 6.2 & 7.6 & 13.6 \\
\hline NML & 14.4 & 7.5 & 7.7 & 5.7 & 7.8 & 15.6 & 9.2 & 9.8 & 6.9 \\
\hline RCA IM 801 & 16.9 & 9.1 & 7.3 & 8.9 & 8.5 & 9.9 & 7.9 & 6.6 & 8.2 \\
\hline RCA IM 921 & 12.0 & 11.4 & 8.1 & 10.7 & 7.4 & 10.1 & 13.9 & 16.9 & 13.3 \\
\hline
\end{tabular}

${ }_{1}$ In four assays the $T_{4}$-concentration was measured in triplicate

$2 \mathrm{~N}=4$

3 Median values per kit are set in italics

Tab. 3. $\mathrm{T}_{4}$ concentrations and ranking numbers per kit for the sera $\mathrm{C} 2-\mathrm{C} 10$. 4 assays in triplicate; $N=12$.

\begin{tabular}{|c|c|c|c|c|c|c|c|c|c|c|c|c|c|c|c|c|c|c|c|}
\hline Kit & C2 & & C3 & & C4 & & C5 & & C6 & & C7 & & & & & & & & \\
\hline & $\mathrm{nmol} / \mathrm{l}$ & no. & $\mathrm{nmol} /$ & no. & $\mathrm{nmol} / 1$ & no. & $\mathrm{nmol} / \mathrm{l}$ & I no. & $\mathrm{nmol}$ & Ino. & $\mathrm{nmol}$ & no. & $\mathrm{nmol} /$ & 1 no. & . $\mathrm{nmol} / \mathrm{l}$ & 1 no. & $\mathrm{nmol} / \mathrm{s}$ & I no. & \\
\hline $\begin{array}{l}\text { Abbott } \\
\text { Becton D } \\
\text { Byk-Riamat } \\
\text { Byk-Spac T } \\
\text { Clinical Assays } \\
\text { Corning } \\
\text { DPC-Premix } \\
\text { Micromedic } \\
\text { NML } \\
\text { RCA-IM } 801 \\
\text { RCA-IM } 921\end{array}$ & $\begin{array}{l}41.8 \\
43.5 \\
28.1 \\
34.2 \\
38.1 \\
54.5 \\
35.5 \\
32.6 \\
50.7 \\
32.5 \\
36.1\end{array}$ & $\begin{array}{r}8 \\
9 \\
1 \\
4 \\
7 \\
11 \\
5 \\
3 \\
10 \\
2 \\
6\end{array}$ & $\begin{array}{l}62.5 \\
65.9 \\
47.0 \\
56.4 \\
59.3 \\
68.3 \\
61.4 \\
54.8 \\
72.8 \\
52.6 \\
44.0\end{array}$ & $\begin{array}{r}8 \\
9 \\
2 \\
5 \\
6 \\
10 \\
7 \\
4 \\
11 \\
3 \\
1\end{array}$ & $\begin{array}{c}117 \\
118 \\
76.3 \\
100 \\
108 \\
104 \\
108 \\
96.3 \\
117 \\
104 \\
77\end{array}$ & $\begin{array}{c}9.5 \\
11 \\
1 \\
4 \\
7.5 \\
5.5 \\
7.5 \\
3 \\
9.5 \\
5.5 \\
2\end{array}$ & $\begin{array}{l}135 \\
139 \\
96.3 \\
118 \\
124 \\
129 \\
128 \\
114 \\
132 \\
124 \\
113\end{array}$ & $\begin{array}{c}10 \\
11 \\
1 \\
4 \\
5.5 \\
8 \\
7 \\
3 \\
9 \\
5.5 \\
2\end{array}$ & $\begin{array}{l}176 \\
180 \\
133 \\
156 \\
160 \\
164 \\
167 \\
146 \\
182 \\
168 \\
157\end{array}$ & $\begin{array}{r}9 \\
10 \\
1 \\
3 \\
5 \\
6 \\
7 \\
2 \\
11 \\
8 \\
4\end{array}$ & $\begin{array}{l}217 \\
220 \\
173 \\
184 \\
205 \\
205 \\
208 \\
186 \\
219 \\
208 \\
213\end{array}$ & $\begin{array}{r}9 \\
11 \\
1 \\
2 \\
4.5 \\
4.5 \\
6.5 \\
3 \\
10 \\
6.5 \\
8\end{array}$ & $\begin{array}{l}264 \\
249 \\
202 \\
225 \\
233 \\
254 \\
250 \\
210 \\
269 \\
222 \\
252\end{array}$ & $\begin{array}{r}10 \\
6 \\
1 \\
4 \\
5 \\
9 \\
7 \\
2 \\
11 \\
3 \\
8\end{array}$ & $\begin{array}{c}298 \\
274 \\
216 \\
248 \\
255 \\
311 \\
>309 \\
246 \\
298 \\
271 \\
251\end{array}$ & $\begin{array}{l}- \\
- \\
- \\
- \\
- \\
- \\
- \\
- \\
- \\
-\end{array}$ & $\begin{array}{l}146 \\
138 \\
104 \\
124 \\
124 \\
139 \\
135 \\
116 \\
137 \\
125 \\
113\end{array}$ & $\begin{array}{r}11 \\
9 \\
1 \\
5 \\
5 \\
10 \\
7 \\
3 \\
8 \\
5 \\
2\end{array}$ & $\begin{array}{l}74.5 \\
76 \\
9 \\
31 \\
45.5 \\
64 \\
54 \\
23 \\
79.5 \\
38.5 \\
33\end{array}$ \\
\hline
\end{tabular}

Tab. 4: Percentage recovery of $T_{4}$ added to serum.

\begin{tabular}{|c|c|c|c|c|}
\hline $\begin{array}{l}\text { Added } \\
\text { Recovered }\end{array}$ & $\begin{array}{l}75 \mathrm{nmol} / 1 \\
\bar{x} \pm \mathrm{SD}^{1}\end{array}$ & $\begin{array}{l}150 \mathrm{nmol} / 1 \\
\bar{x} \pm S D^{1}\end{array}$ & $\begin{array}{l}225 \mathrm{nmol} / 1 \\
\overline{\mathrm{x}} \pm \mathrm{SD}^{1}\end{array}$ & $\begin{array}{l}\text { Total } \\
\bar{x} \pm \mathrm{SD}^{2}\end{array}$ \\
\hline $\begin{array}{l}\text { Abbott } \\
\text { Bectón D } \\
\text { Byk-Riamat } \\
\text { Byk-Spac T4 } \\
\text { Clinical Assays } \\
\text { Corning } \\
\text { DPC-Premix } \\
\text { Micromedic } \\
\text { NML } \\
\text { RCA-IM } 801 \\
\text { RCA-IM } 921\end{array}$ & $\begin{array}{r}107.5 \pm 4.7 \\
109.1 \pm 3.1 \\
88.9 \pm 6.8 \\
98.9 \pm 2.0 \\
101.6 \pm 3.5 \\
107.3 \pm 1.6 \\
111.1 \pm 4.3 \\
108.5 \pm 1.8 \\
121.1 \pm 2.0 \\
103.8 \pm 2.1 \\
95.3 \pm 3.6\end{array}$ & $\begin{array}{rr}105.8 & \pm 3.6 \\
105.4 & \pm 6.6 \\
85.3 & \pm 10.9 \\
98.1 & \pm 2.2 \\
105.2 & \pm 2.8 \\
103.4 & \pm 2.7 \\
117.2 & \pm 8.5 \\
104.8 & \pm 5.4 \\
107.6 & \pm 4.1 \\
98.9 & \pm 1.7 \\
93.3 & \pm 2.7\end{array}$ & $\begin{array}{r}100.4 \pm 2.9 \\
99.9 \pm 5.1 \\
83.6 \pm 8.5 \\
96.2 \pm 2.0 \\
96.0 \pm 1.6 \\
108.2 \pm 1.1 \\
115.2 \pm 5.8 \\
107.0 \pm 3.8 \\
105.9 \pm 4.1 \\
97.8 \pm 2.4 \\
93.3 \pm 1.9\end{array}$ & $\begin{aligned} 104.6 & \pm 4.8 \\
104.8 & \pm 6.2 \\
85.9 & \pm 8.7 \\
97.7 & \pm 2.2 \\
100.9 & \pm 4.7 \\
106.3 & \pm 2.8 \\
114.5 & \pm 6.6 \\
106.8 & \pm 4.0 \\
111.5 & \pm 7.8 \\
100.2 & \pm 3.3 \\
94.0 & \pm 2.8\end{aligned}$ \\
\hline
\end{tabular}

\footnotetext{
$1 \mathrm{~N}=6$

$2 N=18$
} 
between the $T_{4}$-free serum and the zero control samples, except in the case of Becton D and Micromedic. For these two kits the $T_{4}$ concentration of the $T_{4}$-free serum was estimated to be less than $5 \mathrm{nmol} / 1$.

For each kit the time necessary for the processing of 100 tubes was noted. With the exception of incubation times, this included all operations from writing the protocol to calculating the samples from the standard curve. Table 5 shows these times, as well as the number of samples that can be determined in an experiment using 100 tubes.

\section{Discussion}

Probably one of the best ways to show the correlation between the dose level and its response error is the precision profile (3). To construct such a graph from experimental results, however, many dose levels must be investigated. Another possibility is to divide the measuring range into two or three parts and to calculate the average response error. This can be done in several ways, none of which is to be preferred over the others. Therefore, we have decided to present all figures for the nine sera tested.

From table 2 it can be seen that there are differences in the intra- and interassay variations from kit to kit, which

Tab. 5. Labour time for an assay of 100 tubes.

\begin{tabular}{lll}
\hline Kit & $\begin{array}{l}\text { Labour time } \\
(\mathrm{min})\end{array}$ & $\begin{array}{l}\text { Number of } \\
\text { samples }\end{array}$ \\
\hline Abbott & 160 & 45 \\
Becton & 115 & 44 \\
Byk-Riamat & 150 & 43 \\
Byk-Spac T & 115 & 43 \\
Clinical Assays & 115 & 44 \\
Corning & 155 & 45 \\
DPC-Premix & -160 & 44 \\
Micromedic & 145 & 43 \\
NML & 175 & 45 \\
RCA-IM 801 & 175 & 46 \\
RCA-IM 921 & 160 & 46 \\
\hline
\end{tabular}

\section{References}

1. McDonagh, B., Munson, P. J. \& Rodbard, D. (1977),.Comp. Prog. Biomed. 7, 179-190.

2. Thompson, W. A. \& Willke, T. A. (1963), Biometrika 50, $375-380$. are difficult to explain. It has been reported that radioimmunoassays using antibody-coated tubes tend to have higher coefficients of variation (4). Our results (Becton D, SPAC T 4 , Clinical Assays and Micromedic) seem to support this notion. Furthermore, the sample volume may be important. The four kits with a small presented sample volume of $10 \mu \mathrm{l}$ (Becton D, Byk-Riamat, NML and Clinical Assays) show a rather large intra-assay variation, whereas RCA IM801 and RCA IM921 with a large sample volume $(50 \mu \mathrm{l})$ yield comparatively good results.

From tables 3 and 4 it can be seen that certain kits yield systematically lower (Byk-Riamat) or higher (Becton D, $\mathrm{NML}) \mathrm{T}_{4}$ concentrations. Since there is not yet a definitive method for the measurement of $\mathrm{T}_{\mathbf{4}}$, it cannot be stated that the results of these kits are wrong. However, it is necessary that each laboratory determines its own normal values. Moreover, Byk Riamat combines, in comparison with other kits, a low measured level with a low percentage recovery, whereas NML shows exactly the opposite. These observations may be explained either by an incorrect description of the serum standards or by an incomplete separation of $T_{4}$ from $T B G$.

Concerning the labour-time per 100 tubes (tab. 5) it should be noted that all pipetting was carried out with semi-automatic pipettes. Likewise all calculations, drawing the standard curves and reading off the samples were done by hand. By automation the labour-time could be reduced. The results in this table, therefore, should be considered comparatively. As was to be expected, the kits employing an antibody-coated tube were clearly less labour-intensive.

The overall performance of any kit is determined by the way it fits a number of criteria, some of which have been investigated in:this study. It is difficult to recommend or reject any kit in particular because the choice to be made depends on the demands of the investigator.

\section{Acknowledgements}

The authors wish to thank Drs J. H. H. Thisen and A. B. Leussink for their valuable advice.

3. Ekins, R. P. (1978), in: Radioimmunoassay and Related Procedures in Medicine (IAEA, Vienna), Vol. II, 39-56

4. Nocke-Finck, L., Kahrer, R., Horn, I. \& Bremer, H. (1978), Endokrinologie-Informationen 2, 49-63.

L. H. Elvers

National Institute of Public Health P.O. Box 1

NL-3720 BA Bilthoven 